\title{
The analysis of the prestressed concrete beam damage process based on the acoustic emission
}

\author{
Wang You-Shun ${ }^{1, a^{*}, \text { Liu Jian }}{ }^{2, b}$ \\ ${ }^{1}$ Zhumadian Highway Administration Bureau, Zhumadian 463000 ,Henan, China
}

\begin{abstract}
Keywords: AE;prestressed concrete;the damage evaluation;pattern recognition
Abstract. The use of acoustic emission technology of prestressed concrete beams damage monitoring research, obtained the prestressed concrete beam damage acoustic emission monitoring of real-time data; according to the value of the acoustic emission energy parameters of acoustic emission, reveals that the bearing capacity of concrete beams down cause and the damage mechanism; In addition, through calculation of the characteristics of acoustic emission signal Kurtosis index, observe the damage evolution process of prestressed concrete beam, determine the reinforced concrete beam damage stage.Late as well as further on the long-term health of the reinforced concrete structure damage active monitoring to provide theoretical basis.
\end{abstract}

\section{Introduction}

Acoustic emission technique is a non-destructive testing method, which have a unique advantage such as real-time,integrity, high sensitivity, can be dynamic health monitor important engineering structure.Acoustic emission refers to the structure materials unrecoverable damage occurs, local rapid release of energy from the material and produce the phenomenon of transient elastic wave ${ }^{[1]}$.

Along with the progress of $\mathrm{AE}$ instruments, acoustic emission technology rapidly into civil engineering structure detection field, the study of materials and components on different levels ${ }^{[2,3]}$, But on the whole, focused on the damage detection of steel structure and concrete material, the application of concrete structure by $\mathrm{AE}$ is relatively small,And concrete structure safety and service ability has always been a problem that nots allow to ignor health monitoring and damage detection for important engineering structures (such as bridge structure) is extremely important and key components.

Against the above background, this paper, by using AE technology to real-time monitoring loads of prestressed reinforced concrete beam specimen damage conditions, $\mathrm{AE}$ characteristic parameters and damage variable have consistency, acoustic emission signals and types of waves has the close relation ${ }^{[4]}$.Using the correlation analysis of the acoustic emissionmethod of component analysis, the damage evolution process can be qualitative or quantitative assessment of the damage degree of reinforced concrete component damage status ofAE recognition ${ }^{[5,6]}$.

\section{The experimental scheme:}

\section{Experimental beams:}

Specimen size: $150 \mathrm{~mm} \times 250 \mathrm{~mm} \times 1200 \mathrm{mmin}$ accordance with the appropriate reinforcement beam reinforcement,Choice as a steel2B10, handling reinforcement for $2 \mathrm{~B} 10$ using 1x3,prestressed steel strand, 1.5h within the scope of the A8@100, other stirrup the A8@150.
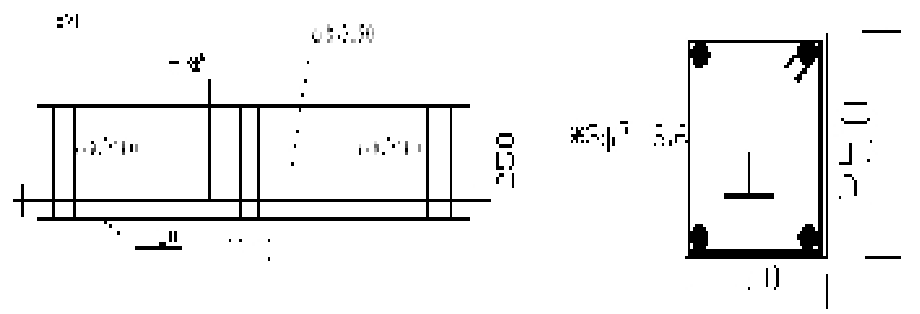

Fig. 1 Prestressed concrete beam loadchart with reinforcement detailing( $\mathrm{mm})$ 


\section{Acoustic emission instrument and parameter Settings :}

Instrument technology using sound China SAEU2S-4 channel USB acoustic emission system, frequency range $1-400 \mathrm{Khz}, 16$ bit $\mathrm{A} / \mathrm{D}$ converter,can collecting waveform and AE parameters.

\begin{tabular}{cccccc}
\hline gain/dB & threshold/dB & filter $/ \mathrm{kHz}$ & PDT/us & HDT/us & HLT/us \\
\hline 40 & 50 & $1-400$ & 220 & 650 & 800 \\
\hline
\end{tabular}

Table 1 Setting of AE parameters

loading ways:

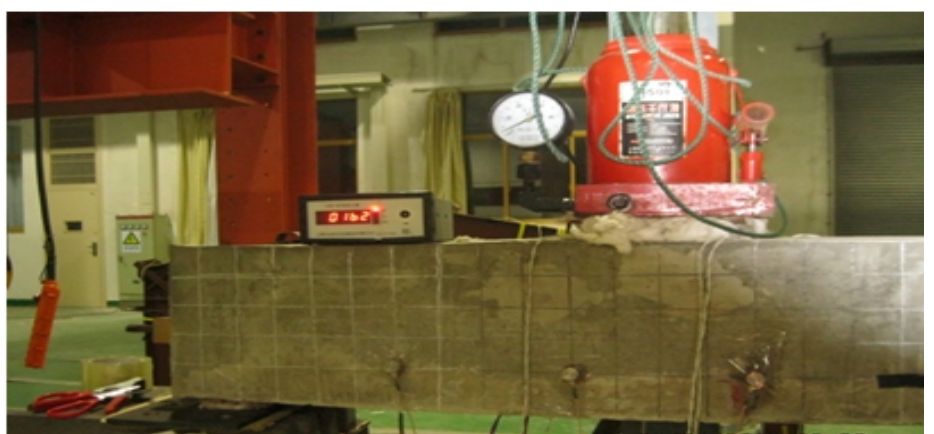

Fig. 2 Specimen size and layout of the sensors

Adopts hydraulic jack for loading equipment, rated lifting weight is 50t, the bearing and load points mat cotton and rubber mat to isolate external noise within the incoming beam. Also ruled out test in the process of mechanical noise interference of acoustic emissionsignals.

\section{The experimental results and analysis:}

AE energy advantage is able to reflect the signal amplitude and duration, and is not sensitive to the signal frequency and voltage threshold ${ }^{[7,8,9]}$.

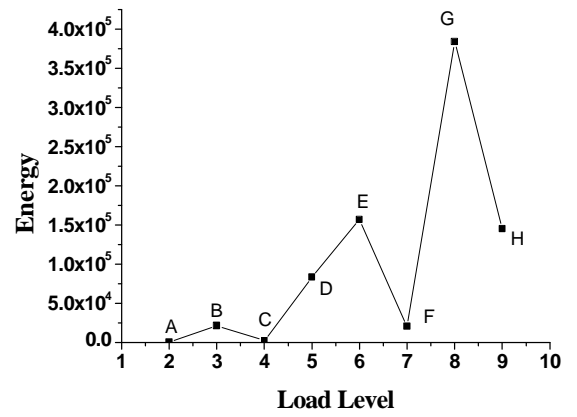

(1) Acoustic emission energy relationship with relations load level

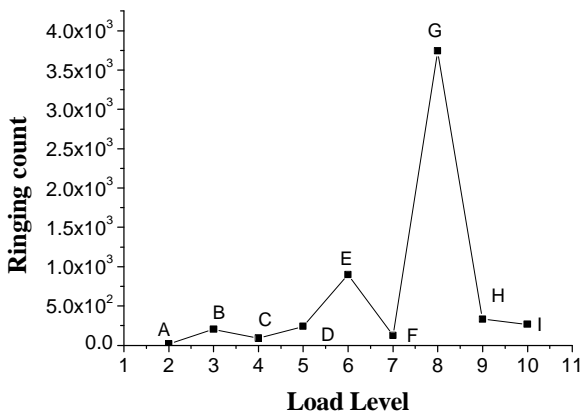

(2) Acoustic emission ringing count with load level

Fig. 3 Changing of each AE parameter with the loading grade

In the slight damage stages of beam, AE events energy levels are smaller, the signal is given priority to with low amplitude of the signal as shown in figure 4 when there is a large energy of event $\mathrm{B}$, micro defects polymerization begin to form micro cracks to a certain degree,show that in the second stage. The beam of microfracture formation stage, AE events become active at this time.

When the load increases to nearly 6 stages, produced by macro cracking $E$ and F.Acoustic emission events $\mathrm{E}$ has high ringing counts, amplitude, thus energy is larger, can be seen at this stage of beam damage hav a certain degree of damage status became more complicated, high amplitude signal began to increase as shown in figure 4, the signal from the concrete interface instability and local slip between steel bar and concrete began to appear.

When the load is close to $75 \% \operatorname{load}(\mathrm{G})$, events energy level increase, high amplitude of the signal number further growth,suggests that further damage, at the same time appearslip between steel bar and reinforced concrete, the component can still work, but the structure reliability decreases obviously, warning signal structure need to be reinforced, repair or do further testing. 
When close to the event $\mathrm{H}, \mathrm{I}$, the components into the failure destruction stage, load limitvalue, overall beam stiffness significantly decreased at the same time,macro fracture development to concrete compressive zone, in the process, damage event $\mathrm{H}$, I produced a large energy release.
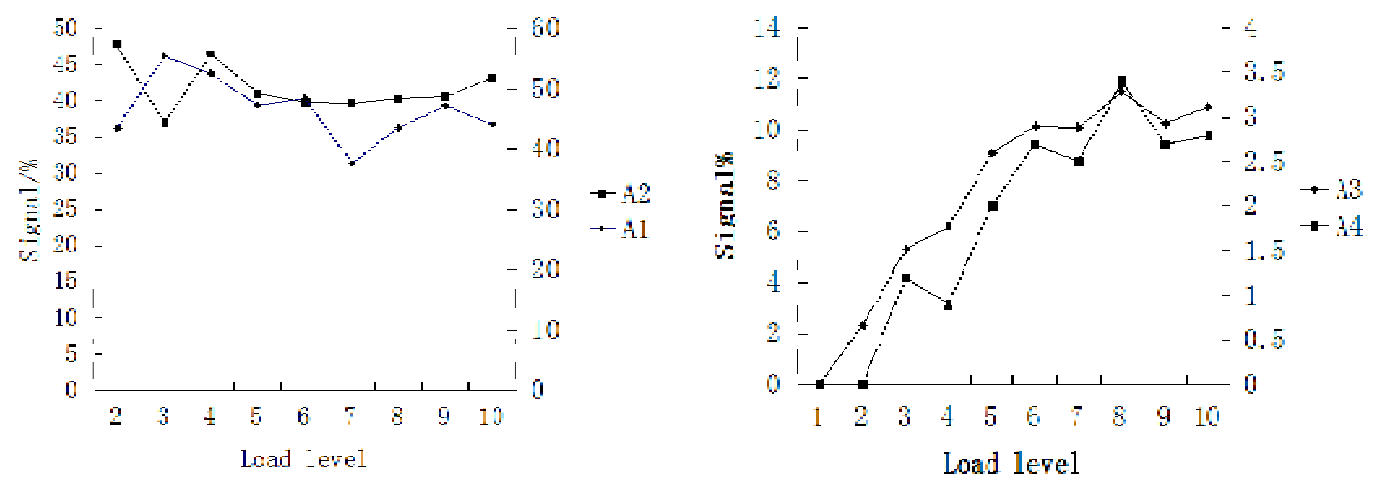

A1 <60db A2 60-70db A3 70-80db A4>80db

Fig. 4 Different signal amplitude percentage change curve

\section{Kurtosis index assessment of prestressed reinforced concrete beam damage evolution:}

Instantaneous indicators are often used to assess the possible structure damage and service ability. We using Kurtosis index analysis AE signal, Kurtosis index calculation method is as follows ${ }^{[10]:}$

$$
\text { Kurtosis }=\frac{\frac{1}{N} \sum_{n=1}^{N}\left(x(n)-\frac{1}{N} \sum_{n=1}^{N} x(n)\right)^{4}}{\left[\frac{1}{N} \sum_{n=1}^{N}\left(x(n)-\frac{1}{N} \sum_{n=1}^{N} x(n)\right)^{2}\right]^{2}}
$$

Formula, $x(n)$ the AE signal amplitude, $N$ time domain signal data number in each group, using the above formula to calculate the damage Kurtosis value of indicators reflect the differences between data, the greater the Kurtosis index, the difference between the set of data, the greater the Kurtosis index is smaller, the smaller the difference between the data, the damage by the weaker,at this time of $\mathrm{N}$ for each loading cycle, AE signal stronger, the greater the Kurtosis value also, at the sametime, the greater the damage degree, this experiment, will load as a group, each calculation of Kurtosis index.
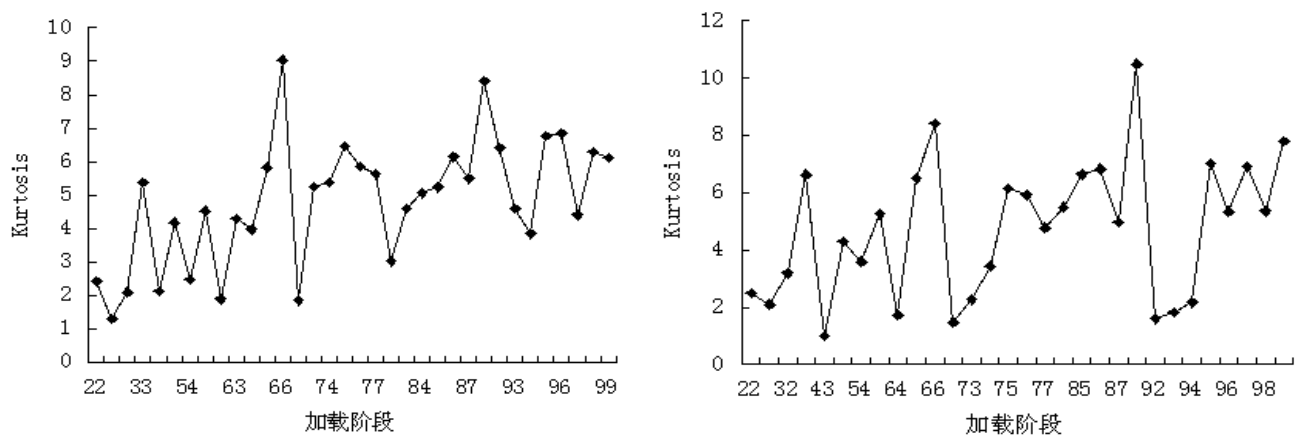

Fig.5 Kurtosis index curve ( 4 channel) Fig.6 Kurtosis index curve ( 3 channel)

Kurtosis parameter values as shown in figure 5-6, in 88 and 66 before and after loading, extremum Kurtosis index, internal injury is serious, the extreme value point in time in conformity with the front level analysis, evolution curve can be observed extreme value point to determine the degree of damage of reinforced concrete beam, we can determine the beam damage four typical failure phase:

(1) The mesoscopic damage polymerization phase (1 load to load level 3) : the early loading, prestressed reinforced concrete beam of prestressed steel wire tension, under offset load effectively, basic no AE signal, when the load increases further, material internal force deformation caused by the micro defects (such as holes, etc.), closed emarginated defects gradually compressed at the same time, the acoustic emission signal occur. 
(2) Mesoscopic crack development stage (3 loaded into 6 loading level) : micro crackinitiation stage, is also a mesoscopic damage stages of development. Micro cracks will further extend under the point of stress concentration, cracks activities to strengthen performance, under the condition of the crack in the load is no longer increasing will not continue to expand, part of the crack can be closed when unloading.

(3) Stability of crack extension stage (load 6 to 8 loading level) : continue to load until this stage of the reinforced concrete beam about $75 \%$ damage development gradually expanded. crack at the macro level, the corresponding stress - strain curve at this time also deviate from the linear,,at the same time, this phase will produce more new micro cracks, and these will be stable extension of crack combined into macroscopic crack .

(4) The stage of crack instability propagation(8 loading level to fail) : when loading force near the critical load, the damage evolution of accelerated dramatically, internal continuous crack formation, a large number of cracks. Acoustic emission signal will increase suddenly, acoustic emission counts curve exponential growth, internal injury is serious.

\section{Conclusion:}

In this paper, the load damage process of prestressed reinforced concrete beam under the action of the acoustic emission detection and analysis. Main conclusions are as follows:

Acoustic emission signals and damage of prestressed reinforced concrete has good correlation, can accurately track the reinforced concrete beam damage characteristics of each stage;

with the help of energy curve and Kurtosis index, beam damage evolution process in specific analysis, concluded four typical failure stages: mesoscopic damage aggregation stage, the mesoscopic crack development stage, the stability of crack extension stage, stage of crack instability propagation;

\section{References}

[1] Beattie A G, Acoustic emission, principles and instrumentation [J].Journal of Acoustic Emission, 1983, 2(1/2): 95 -128;

[2] Eberhartdt E,Stead D,Stimpson B,etal Changes in acoustic event properties with progressive fracture damage[J] .Int.J RockMech \& Min.Sic.1997(34):3-4;

[3] Nair Archana ,Cai C S .Acoustic emission monitoring of bridges:review and case studies [J].Engine ering Structure.2010(32):1704-1714;

[4] Zhang M,Li ZK,Yang Qetc,Quasi brittle material damage model of acoustic emission and statistical analysis[J],Journal of rock mechanics and engineering,2006(25):2493-2501;

[5] Zhu HP,Xu WS,Chen XQ,etc,Using the acoustic emission signal and quantitative evaluation of concrete damage rate process theory[J],Engineering mechanics,2008(1):186-191;

[6] Kim B,Weiss W J,Using acoustic emission to quantify damage in restrained fiber-reinforced cement mortars[J].Cement and Concrete Research ,2003(3):207-214;

[7] Han W Q,Zhou J Y,Acoustic emission characterization methods of damage modes identification on carbon fiber twill weave laminate[J],Science china-Technological Sciences,2013:2228-2237;

[8] Hamdi S E,Le Duff A,Simon L,et al.Acoustic emission pattern recognition approach based on Hilber t-Huang transform for structural health monitoring in polymer-composite materials[J],Applied Acoustic, 2013.74(5):764-757;

[9] Gu AJ,Reinforced concrete members damage status of ae recognition.Journal of huaiyin institute of technology,2015(2):42-45 
[10]Chiementin X,Mba D,Charnley B,Effect of the Denoising on Acoustic Emission signals[J],Journal of Vibration and Acoustics,2010,132(3), : 1-9; 